\title{
Angewandte
}

Supporting Information

(C) Wiley-VCH 2011

69451 Weinheim, Germany

\section{Zwitterionic Si-C-Si-P and Si-P-Si-P Four-Membered Rings with Two-Coordinate Phosphorus Atoms**}

Sakya S. Sen, Shabana Khan, Herbert W. Roesky, * Daniel Kratzert, Kathrin Meindl, Julian Henn, Dietmar Stalke, * Jean-Philippe Demers, and Adam Lange

anie_201005866_sm_miscellaneous_information.pdf 


\section{Content:}

(S1). Syntheses of $\mathbf{3}$ and $\mathbf{4}$

(S2). Crystal data for $\mathbf{3}$ and $\mathbf{4}$

(S3). Theoretical Calculation for $\mathbf{3}$ and $\mathbf{4}$

(S4). Solid-state NMR

(S5). Probable mechanism for the formation of $\mathbf{3}$ and $\mathbf{4}$ from 2.

(S6). References

\section{(S1). Syntheses of 3 and 4}

All reactions and handling of reagents were performed under an atmosphere of dry nitrogen or argon using standard Schlenk techniques or a glove box where the $\mathrm{O}_{2}$ and $\mathrm{H}_{2} \mathrm{O}$ levels were usually kept below $1 \mathrm{ppm}$. Adamantyl phosphalkyne was purchased from Aldrich and used as received. White phosphorus was sublimized prior to use. Compounds $\mathbf{1}$ and $\mathbf{2}$ were prepared according to literature methods. ${ }^{\left[{ }^{[1, S 2]}\right.}$ Solvents were purified with the M-Braun solvent drying system. Solution NMR spectra were recorded on Bruker Avance 200, Bruker Avance 300, and Bruker Avance $500 \mathrm{MHz}$ NMR spectrometers. Deuterated NMR solvents $\mathrm{C}_{6} \mathrm{D}_{6}$, and $\mathrm{D}_{8^{-}}$ [THF] were dried by stirring for 2 days over $\mathrm{Na} / \mathrm{K}$ alloy followed by distillation in vacuum and degassed. EI-MS spectra were obtained with a Finnigan MAT 8230 or a Varian MAT CH5 instrument $(70 \mathrm{eV})$ by EI-MS methods. Elemental analyses were performed by the Analytisches Labor des Instituts für Anorganische Chemie der Universität Göttingen. Melting points were measured in sealed glass tubes on a Büchi B-540 melting point apparatus.

3: To the mixture of $1(0.29 \mathrm{~g}, 1.00 \mathrm{mmol})$ and 1-adamantyl phosphaalkyne $(0.07 \mathrm{~g}, 0.38$ mmol) at $-20{ }^{\circ} \mathrm{C}$ toluene $(25 \mathrm{~mL})$ was added and the color of the solution immediately 
changed from colorless to red. The reaction mixture was stirred overnight. The solvent was removed under reduced pressure and the crude product was extracted with toluene $(10 \mathrm{~mL})$. Concentration and storing at $0{ }^{\circ} \mathrm{C}$ in a freezer afforded reddish yellow crystals of $\mathbf{3}(0.15 \mathrm{~g}$, 68.22\%). ${ }^{1} \mathrm{H}$ NMR (200 MHz, $\left.\mathrm{C}_{6} \mathrm{D}_{6}, 25^{\circ} \mathrm{C}\right): \delta 1.21$ (s, 4H, Ad), 1.26 (s, $\left.36 \mathrm{H}, t \mathrm{Bu}\right), 1.72$ (s, $12 \mathrm{H}, \mathrm{Ad}), 6.90-7.10(\mathrm{~m}, 10 \mathrm{H}, \mathrm{Ph}) \mathrm{ppm} ;{ }^{13} \mathrm{C} \mathrm{NMR}\left(125.75 \mathrm{MHz}, \mathrm{C}_{6} \mathrm{D}_{6}, 25{ }^{\circ} \mathrm{C}\right): \delta 32.8$ $\left(\mathrm{CMe}_{3}\right), 40.2$, 41.6, 50.9, 51.6 (adamantyl), $56.9\left(\mathrm{CMe}_{3}\right), 129.4,130.6,132.2,133.2(P h)$, $136.2\left(\mathrm{Si}-C\right.$-Ad), $171.23(\mathrm{NCN}) \mathrm{ppm} ;{ }^{31} \mathrm{P} \mathrm{NMR}\left(121.5 \mathrm{MHz}, \mathrm{C}_{6} \mathrm{D}_{6}, 25{ }^{\circ} \mathrm{C}\right)-243 \mathrm{ppm}$; ${ }^{29} \mathrm{Si}\left\{{ }^{1} \mathrm{H}\right\}$ NMR $\left(99.36 \mathrm{MHz}, \mathrm{C}_{6} \mathrm{D}_{6}, 25{ }^{\circ} \mathrm{C}\right): \delta-5.1 \mathrm{ppm}\left({ }^{1} J_{\mathrm{Si}-\mathrm{P}}=75.45 \mathrm{~Hz}\right)$; EI-MS: $m / z: 696$ (100\%). Elemental analysis for $\mathrm{C}_{41} \mathrm{H}_{61} \mathrm{~N}_{4} \mathrm{PSi}_{2}$ : calcd $\mathrm{C}, 70.64 ; \mathrm{H}, 8.82 ; \mathrm{N}, 8.04$; found $\mathrm{C}$, $71.16 ; \mathrm{H}, 9.12 ; \mathrm{N}, 8.10$.

Alternate synthesis of 3: To the mixture of $2(0.10 \mathrm{~g}, 0.19 \mathrm{mmol})$ and 1-adamantyl phosphaalkyne $(0.03 \mathrm{~g}, 0.19 \mathrm{mmol})$ at $-20{ }^{\circ} \mathrm{C}$ toluene $(25 \mathrm{~mL})$ was added and the color of the solution immediately changed from dark red to pale red. The reaction mixture was stirred overnight. Concentration and storing at room temperature afforded reddish yellow crystals of $3(0.06 \mathrm{~g}, 44.78 \%)$.

4: To the mixture of $1(0.29 \mathrm{~g}, 1.00 \mathrm{mmol})$ and white phosphorus $(0.124 \mathrm{~g}, 1.00 \mathrm{mmol})$ at room temperature toluene $(25 \mathrm{~mL})$ was added and the color of the solution immediately changed from colorless to dark yellow with the formation of a yellow solid. The reaction mixture was stirred overnight. The solvent was removed under reduced pressure, the solid was washed with toluene $(10 \mathrm{~mL})$ to remove $\mathrm{PhC}(\mathrm{N} t \mathrm{Bu})_{2} \mathrm{SiCl}_{3}$ and then extracted with THF (25 $\mathrm{mL})$. Concentration and storing at $-32{ }^{\circ} \mathrm{C}$ in a freezer afforded yellow crystals of $4(0.17 \mathrm{~g}$, 60\%). Mp 168-175 ${ }^{\circ} \mathrm{C} .{ }^{1} \mathrm{H}$ NMR (500 MHz, [D 8 -THF, $\left.25^{\circ} \mathrm{C}\right): \delta 1.36$ (s, $\left.36 \mathrm{H}, t \mathrm{Bu}\right), 6.61-$ $6.98(\mathrm{~m}, 10 \mathrm{H}, \mathrm{Ph}) \mathrm{ppm} ;{ }^{13} \mathrm{C}\left\{{ }^{1} \mathrm{H}\right\}$ NMR $\left(125.75 \mathrm{MHz},\left[\mathrm{D}_{8}\right]-\mathrm{THF}, 25{ }^{\circ} \mathrm{C}\right): \delta 33.1\left(\mathrm{CMe}_{3}\right), 53.5$ $\left(C \mathrm{Me}_{3}\right), 125.6,127.6,129.3,135.9(\mathrm{Ph}), 171.23(\mathrm{NCN}) \mathrm{ppm} ;{ }^{31} \mathrm{P}$ NMR $\left(121.5 \mathrm{MHz},\left[\mathrm{D}_{8}\right]-\right.$ 
THF, $\left.25{ }^{\circ} \mathrm{C}\right): d-166.02 \mathrm{ppm} ;{ }^{29} \mathrm{Si}\left\{{ }^{1} \mathrm{H}\right\}$ NMR $\left(99.36 \mathrm{MHz},\left[\mathrm{D}_{8}\right]-\mathrm{THF}, 25^{\circ} \mathrm{C}\right): d 25.6 \mathrm{ppm}$ $\left({ }^{1} J_{\text {Si-P }}=109.02 \mathrm{~Hz}\right)$. EI-MS: $m / z: 580\left[\mathrm{M}^{+}\right](100 \%)$. Elemental analysis for $\mathrm{C}_{30} \mathrm{H}_{46} \mathrm{~N}_{4} \mathrm{P}_{2} \mathrm{Si}_{2}$ : calcd C, 62.04; H, 7.98; N, 9.65; found C, 62.29; H, 8.08; N, 9.72.

Alternate synthesis of 4 : THF $(25 \mathrm{~mL})$ was charged to the mixture of white phosphorus $(0.03 \mathrm{~g}, 0.21 \mathrm{mmol})$ and $2(0.11 \mathrm{~g}, 0.21 \mathrm{mmol})$ at room temperature. The color of the solution changed immediately from deep red to faint red. The reaction mixture was stirred for $2 \mathrm{~h}$ and all volatiles were removed in vacuum. The residue was treated with THF (10 mL) and filtered through celite. The solution was concentrated and stored at $-32{ }^{\circ} \mathrm{C}$ to yield yellow crystals of $4(0.12 \mathrm{~g}, 50 \%)$

\section{(S2). Crystal data for 3 and 4}

Shock cooled crystals were selected and mounted under nitrogen atmosphere using the XTEMP2. ${ }^{[\mathrm{S} 3]}$ The data of $\mathbf{3}$ was measured on a Bruker TXS-Mo rotating anode with Helios mirror optics; 4 was measured on an INCOATEC Mo Microsource with Quazar mirror optics. Both had a APEX II detector on a D8 goniometer. The diffractometers where equipped with a low-temperature device and used $\mathrm{MoK}_{\mathrm{a}}$ radiation, ? = $0.71073 \AA$. The data sets were integrated with SAINT $^{[\mathrm{S} 4]}$ and an empirical absorption (SADABS) was applied. ${ }^{[\mathrm{S} 5]}$ The structures were solved by direct methods (SHELXS-97) and refined by full-matrix leastsquares methods against $F^{2}$ (SHELXL-97). ${ }^{[\mathrm{S} 6]}$ All non-hydrogen-atoms were refined with anisotropic displacement parameters. The hydrogen atoms were refined isotropically on calculated positions using a riding model with their $\mathrm{U}_{\text {iso }}$ values constrained to equal to 1.5 times the $\mathrm{U}_{\mathrm{eq}}$ of their pivot atoms for terminal $\mathrm{sp}^{3}$ carbon atoms and 1.2 times for all other carbon atoms. Disordered moieties were refined using bond lengths restraints and isotropic displacement parameters restraints. Crystallographic data (excluding structure factors) for the structures reported in this paper have been deposited with the Cambridge Crystallographic 
Data Center. The crystal data are available from The Cambridge Crystallographic Data Centre via www.ccdc.cam.ac.uk/data request/cif.

\section{CCDC number}

Empirical formula

Formula weight

Temperature $[\mathrm{K}]$

Wavelength $[\AA]$

Crystal system

Space group

Unit cell dimensions $[\AA],\left[^{\circ}\right]$

Volume $\left[\AA^{3}\right]$

$Z$

Density (calculated) $[\mathrm{Mg} / \mathrm{m}]$

Absorption coefficient $\left[\mathrm{mm}^{-1}\right]$

$F(000)$

Crystal size $\left[\mathrm{mm}^{3}\right]$

Theta range for data collection $\left[{ }^{\circ}\right]$

Index ranges

Reflections collected

Independent reflections

Completeness to theta $=27.13^{\circ}$

Absorption correction

Max. and min. transmission

Refinement method

Data / restraints / parameters

Goodness-of-fit on $F^{2}$

Final $\mathrm{R}$ indices $[\mathrm{I}>2 \operatorname{sigma}(\mathrm{I})]$

$\mathrm{R}$ indices (all data)

Largest diff. peak and hole $\left[\mathrm{e}^{-3}\right]$

3.3toluene
793275
$\mathrm{C}_{62} \mathrm{H}_{85} \mathrm{~N}_{4} \mathrm{PSi}_{2}$
973.49
$100(2)$
0.71073
Orthorhombic
$P 2_{1} 2_{1} 2_{1}$
$a=13.540(2)$
$b=18.636(3)$
$c=22.541(4)$
$5687.8(17)$
4
1.137
0.132
2112
$0.256 \times 0.138 \times 0.10$
1.42 to 27.13
$-17<=h<=17,-23<=k<=23$,
$-28<=l<=28$
84501
$12583[R($ int $)=0.0341]$
$99.9 \%$
$S 6$

Semi-empirical from equivalents 0.98279 and 0.91086

Full-matrix least-squares on $F^{2}$

12583 / $228 / 720$

1.086

$R 1=0.0467, w R 2=0.1240$

$R 1=0.0505, w R 2=0.1267$

0.686 and -0.271

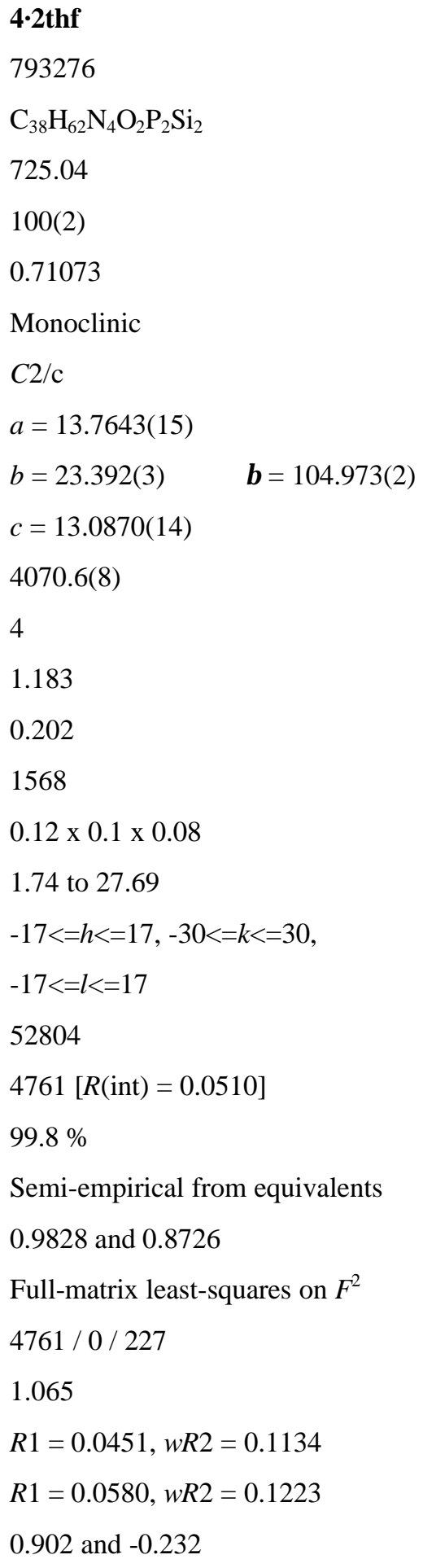


(S3). Theoretical Calculations for 3 and 4

All theoretical calculations were performed with Gaussian 09 (Rev. A.02) on the B3LYP/6-311G* level of theory. ${ }^{[\mathrm{S} 7]}$

Coordinates of the optimized geometry of $\mathbf{3}$

\begin{tabular}{|c|c|c|c|}
\hline N1 & -2.829836 & -0.776811 & -1.047294 \\
\hline $\mathrm{N} 2$ & -2.824241 & -0.647476 & 1.105392 \\
\hline N3 & 2.806978 & -0.662134 & 1.114555 \\
\hline N4 & 2.808124 & -0.826327 & -1.034851 \\
\hline P1 & -0.023152 & -2.599586 & 0.148800 \\
\hline SI1 & -1.259368 & -0.767081 & 0.029421 \\
\hline SI2 & 1.239878 & -0.786597 & 0.037128 \\
\hline $\mathrm{C} 1$ & 5.090299 & -0.829485 & 0.041074 \\
\hline $\mathrm{C} 2$ & 5.825480 & 0.358985 & -0.016939 \\
\hline $\mathrm{H} 2$ & 5.306829 & 1.310472 & -0.058519 \\
\hline C3 & 7.217273 & 0.323837 & -0.038795 \\
\hline $\mathrm{H} 3$ & 7.777119 & 1.252028 & -0.086641 \\
\hline $\mathrm{C} 4$ & 7.887312 & -0.897450 & -0.004110 \\
\hline $\mathrm{H} 4$ & 8.971837 & -0.924290 & -0.022826 \\
\hline C5 & 7.159601 & -2.084164 & 0.055653 \\
\hline H5 & 7.674755 & -3.038574 & 0.086167 \\
\hline C6 & 5.767280 & -2.052343 & 0.079074 \\
\hline H6 & 5.205819 & -2.977910 & 0.137157 \\
\hline C7 & 3.110374 & -1.150439 & -2.450830 \\
\hline $\mathrm{C} 8$ & 4.281231 & -0.321133 & -3.005695 \\
\hline H8A & 5.239339 & -0.597317 & -2.567072 \\
\hline H8B & 4.117486 & 0.746056 & -2.836019 \\
\hline $\mathrm{H} 8 \mathrm{C}$ & 4.355599 & -0.481025 & -4.084884 \\
\hline C9 & 3.397229 & -2.657454 & -2.595692 \\
\hline H9A & 2.573377 & -3.242526 & -2.181305 \\
\hline H9B & 4.319227 & -2.940220 & -2.083932 \\
\hline $\mathrm{H} 9 \mathrm{C}$ & 3.510728 & -2.923124 & -3.650953 \\
\hline C10 & 1.845855 & -0.809575 & -3.250926 \\
\hline $\mathrm{H} 10 \mathrm{~A}$ & 1.604871 & 0.252021 & -3.174986 \\
\hline H10B & 0.993762 & -1.380819 & -2.883515 \\
\hline $\mathrm{H} 10 \mathrm{C}$ & 1.995650 & -1.055829 & -4.305248 \\
\hline C11 & 3.598247 & -0.779351 & 0.042890 \\
\hline $\mathrm{C} 12$ & 3.134279 & -0.570742 & 2.559048 \\
\hline C13 & 1.797752 & -0.628982 & 3.311343 \\
\hline H13A & 1.284056 & -1.571382 & 3.114193 \\
\hline H13B & 1.137591 & 0.184196 & 3.007893 \\
\hline H13C & 1.972102 & -0.547184 & 4.387222 \\
\hline C14 & 3.835790 & 0.761481 & 2.880994 \\
\hline H14A & 3.232147 & 1.606950 & 2.542544 \\
\hline H14B & 4.818895 & 0.827874 & 2.412744 \\
\hline $\mathrm{H} 14 \mathrm{C}$ & 3.977308 & 0.859080 & 3.961211 \\
\hline C15 & 3.999714 & -1.758140 & 3.020568 \\
\hline H15A & 5.005744 & -1.727869 & 2.602391 \\
\hline H15B & 3.531737 & -2.704434 & 2.738726 \\
\hline $\mathrm{H} 15 \mathrm{C}$ & 4.095609 & -1.741595 & 4.109825 \\
\hline C16 & -3.617911 & -0.745188 & 0.030960 \\
\hline $\mathrm{C} 17$ & -5.110592 & -0.792899 & 0.041314 \\
\hline C18 & -5.790447 & -2.014308 & 0.070705 \\
\hline H18 & -5.230834 & -2.942748 & 0.073474 \\
\hline C19 & -7.183179 & -2.042418 & 0.090771 \\
\hline H19 & -7.699721 & -2.996244 & 0.114830 \\
\hline $\mathrm{C} 20$ & -7.908688 & -0.853283 & 0.078104 \\
\hline
\end{tabular}




\begin{tabular}{|c|c|c|c|}
\hline $\mathrm{H} 20$ & -8.993343 & -0.877061 & 0.092690 \\
\hline $\mathrm{C} 21$ & -7.236112 & 0.366844 & 0.046332 \\
\hline $\mathrm{H} 21$ & -7.794121 & 1.297309 & 0.036686 \\
\hline $\mathrm{C} 22$ & -5.844359 & 0.398040 & 0.029878 \\
\hline $\mathrm{H} 22$ & -5.323848 & 1.349263 & 0.013481 \\
\hline $\mathrm{C} 23$ & -3.160000 & -0.865331 & -2.491767 \\
\hline C24 & -1.820502 & -0.887114 & -3.240242 \\
\hline $\mathrm{H} 24 \mathrm{~A}$ & -1.238441 & -1.765965 & -2.957533 \\
\hline H24B & -1.226909 & -0.000971 & -3.013588 \\
\hline $\mathrm{H} 24 \mathrm{C}$ & -1.996231 & -0.921422 & -4.318410 \\
\hline $\mathrm{C} 25$ & -3.912485 & -2.170708 & -2.812093 \\
\hline $\mathrm{H} 25 \mathrm{~A}$ & -4.917169 & -2.183054 & -2.389274 \\
\hline H25B & -3.360110 & -3.032497 & -2.430252 \\
\hline $\mathrm{H} 25 \mathrm{C}$ & -4.010106 & -2.284773 & -3.895498 \\
\hline C26 & -3.977339 & 0.354356 & -2.954144 \\
\hline $\mathrm{H} 26 \mathrm{~A}$ & -3.457548 & 1.283260 & -2.709859 \\
\hline H26B & -4.967534 & 0.381348 & -2.497685 \\
\hline $\mathrm{H} 26 \mathrm{C}$ & -4.115334 & 0.319374 & -4.038571 \\
\hline C27 & -3.128425 & -0.822121 & 2.548471 \\
\hline $\mathrm{C} 28$ & -4.212346 & 0.157340 & 3.031315 \\
\hline $\mathrm{H} 28 \mathrm{~A}$ & -5.194878 & -0.065745 & 2.616478 \\
\hline H28B & -3.951106 & 1.185492 & 2.767545 \\
\hline $\mathrm{H} 28 \mathrm{C}$ & -4.294346 & 0.101007 & 4.120392 \\
\hline C29 & -3.544315 & -2.277120 & 2.838682 \\
\hline H29A & -2.780915 & -2.969500 & 2.477225 \\
\hline H29B & -4.497118 & -2.526575 & 2.368673 \\
\hline $\mathrm{H} 29 \mathrm{C}$ & -3.659272 & -2.428892 & 3.916024 \\
\hline C30 & -1.825595 & -0.526602 & 3.302793 \\
\hline $\mathrm{H} 30 \mathrm{~A}$ & -1.493012 & 0.497610 & 3.128826 \\
\hline H30B & -1.034186 & -1.204409 & 2.981444 \\
\hline $\mathrm{H} 30 \mathrm{C}$ & -1.979096 & -0.661596 & 4.376456 \\
\hline C31 & 0.001327 & 0.506179 & -0.072972 \\
\hline C32 & 0.020513 & 2.024450 & -0.119977 \\
\hline C33 & 1.337702 & 2.555426 & -0.747021 \\
\hline H33A & 2.191387 & 2.185210 & -0.165349 \\
\hline H33B & 1.447759 & 2.145354 & -1.757060 \\
\hline C34 & -1.149173 & 2.586816 & -0.971583 \\
\hline $\mathrm{H} 34 \mathrm{~A}$ & -1.082297 & 2.176567 & -1.985245 \\
\hline H34B & -2.101147 & 2.237254 & -0.552786 \\
\hline C35 & -0.103861 & 2.653518 & 1.297944 \\
\hline H35A & 0.711241 & 2.280225 & 1.928038 \\
\hline H35B & -1.036071 & 2.306675 & 1.757757 \\
\hline C36 & -0.078746 & 4.194444 & 1.254703 \\
\hline H36 & -0.166088 & 4.592415 & 2.273678 \\
\hline C37 & 1.247177 & 4.665254 & 0.627166 \\
\hline H37A & 1.289935 & 5.761498 & 0.603261 \\
\hline H37B & 2.093162 & 4.331076 & 1.240855 \\
\hline C38 & 1.368829 & 4.095677 & -0.798478 \\
\hline H38 & 2.314361 & 4.426534 & -1.246897 \\
\hline C39 & 0.190319 & 4.602292 & -1.651978 \\
\hline H39A & 0.278427 & 4.224909 & -2.678259 \\
\hline H39B & 0.210333 & 5.697514 & -1.716107 \\
\hline C40 & -1.134922 & 4.127902 & -1.025415 \\
\hline $\mathrm{H} 40$ & -1.976250 & 4.479780 & -1.636068 \\
\hline C41 & -1.256963 & 4.698270 & 0.400011 \\
\hline H41A & -1.265948 & 5.795195 & 0.370541 \\
\hline H41B & -2.207991 & 4.387998 & 0.850804 \\
\hline
\end{tabular}


Coordinates of the optimized geometry of $\mathbf{4}$

\begin{tabular}{|c|c|c|c|}
\hline N1 & 2.854412 & 1.077265 & 0.051541 \\
\hline N2 & -2.854399 & 1.077278 & -0.051519 \\
\hline P3 & 0.000000 & -0.000493 & -1.772599 \\
\hline SI1 & 1.300291 & -0.000005 & -0.000002 \\
\hline SI2 & -1.300255 & -0.000003 & -0.000002 \\
\hline $\mathrm{C} 1$ & 3.147983 & 2.485359 & 0.421462 \\
\hline $\mathrm{C} 2$ & 4.317534 & 3.067206 & -0.390612 \\
\hline $\mathrm{H} 2 \mathrm{~A}$ & 5.275854 & 2.619072 & -0.131146 \\
\hline $\mathrm{H} 2 \mathrm{~B}$ & 4.392158 & 4.140150 & -0.193742 \\
\hline $\mathrm{H} 2 \mathrm{C}$ & 4.150666 & 2.934482 & -1.462399 \\
\hline C3 & 3.436916 & 2.581262 & 1.931285 \\
\hline $\mathrm{H} 3 \mathrm{~A}$ & 2.611163 & 2.157588 & 2.506964 \\
\hline H3B & 3.559931 & 3.626724 & 2.228989 \\
\hline $\mathrm{H} 3 \mathrm{C}$ & 4.355212 & 2.052381 & 2.196066 \\
\hline $\mathrm{C} 4$ & 1.880877 & 3.289742 & 0.097584 \\
\hline $\mathrm{H} 4 \mathrm{~A}$ & 1.629233 & 3.213704 & -0.962026 \\
\hline $\mathrm{H} 4 \mathrm{~B}$ & 2.037306 & 4.343290 & 0.342538 \\
\hline $\mathrm{H} 4 \mathrm{C}$ & 1.032561 & 2.927771 & 0.679681 \\
\hline $\mathrm{C} 5$ & 3.646736 & 0.000000 & -0.000001 \\
\hline C6 & 5.138198 & -0.000001 & -0.000003 \\
\hline $\mathrm{C} 7$ & 5.842873 & -0.121136 & 1.201567 \\
\hline $\mathrm{H7}$ & 5.301769 & -0.228530 & 2.135128 \\
\hline $\mathrm{C} 8$ & 7.235346 & -0.117749 & 1.199888 \\
\hline H8 & 7.773545 & -0.211594 & 2.137278 \\
\hline $\mathrm{C} 9$ & 7.934236 & -0.000006 & -0.000005 \\
\hline $\mathrm{H} 9$ & 9.019236 & -0.000008 & -0.000006 \\
\hline $\mathrm{C} 10$ & -3.147983 & 2.485335 & -0.421619 \\
\hline C11 & -1.880765 & 3.289729 & -0.098182 \\
\hline H11A & -1.628767 & 3.213717 & 0.961344 \\
\hline H11B & -2.037288 & 4.343274 & -0.343099 \\
\hline $\mathrm{H} 11 \mathrm{C}$ & -1.032624 & 2.927783 & -0.680551 \\
\hline $\mathrm{C} 12$ & -4.317278 & 3.067317 & 0.390722 \\
\hline $\mathrm{H} 12 \mathrm{~A}$ & -5.275642 & 2.618977 & 0.131800 \\
\hline H12B & -4.392130 & 4.140186 & 0.193522 \\
\hline $\mathrm{H} 12 \mathrm{C}$ & -4.149925 & 2.934947 & 1.462474 \\
\hline C13 & -3.437290 & 2.581038 & -1.931367 \\
\hline H13A & -2.611784 & 2.157053 & -2.507186 \\
\hline H13B & -3.560092 & 3.626479 & -2.229233 \\
\hline $\mathrm{H} 13 \mathrm{C}$ & -4.355786 & 2.052367 & -2.195844 \\
\hline C14 & -3.646724 & 0.000006 & -0.000005 \\
\hline $\mathrm{C} 15$ & -5.138191 & 0.000002 & -0.000011 \\
\hline C16 & -5.842864 & -0.121265 & -1.201568 \\
\hline H16 & -5.301769 & -0.228785 & -2.135119 \\
\hline C17 & -7.235338 & -0.117879 & -1.199893 \\
\hline H17 & -7.773528 & -0.211817 & -2.137279 \\
\hline C18 & -7.934236 & -0.000013 & -0.000017 \\
\hline H18 & -9.019237 & -0.000019 & -0.000019 \\
\hline N1A & 2.854416 & -1.077269 & -0.051538 \\
\hline $\mathrm{N} 2 \mathrm{~A}$ & -2.854407 & -1.077269 & 0.051527 \\
\hline P3A & -0.000001 & 0.000480 & 1.772595 \\
\hline $\mathrm{C} 1 \mathrm{~A}$ & 3.147997 & -2.485361 & -0.421458 \\
\hline $\mathrm{C} 2 \mathrm{~A}$ & 4.317558 & -3.067192 & 0.390611 \\
\hline $\mathrm{H} 2 \mathrm{AA}$ & 5.275871 & -2.619043 & 0.131144 \\
\hline $\mathrm{H} 2 \mathrm{BA}$ & 4.392197 & -4.140135 & 0.193740 \\
\hline $\mathrm{H} 2 \mathrm{CA}$ & 4.150692 & -2.934473 & 1.462400 \\
\hline $\mathrm{C} 3 \mathrm{~A}$ & 3.436926 & -2.581264 & -1.931282 \\
\hline H3AA & 2.611167 & -2.157600 & -2.506959 \\
\hline H3BA & 3.559954 & -3.626724 & -2.228984 \\
\hline H3CA & 4.355216 & -2.052371 & -2.196066 \\
\hline
\end{tabular}




\begin{tabular}{l|rrr} 
C4A & 1.880902 & -3.289759 & -0.097575 \\
H4AA & 1.629258 & -3.213722 & 0.962035 \\
H4BA & 2.037343 & -4.343306 & -0.342528 \\
H4CA & 1.032581 & -2.927800 & -0.679672 \\
C7A & 5.842872 & 0.121130 & -1.201574 \\
H7A & 5.301767 & 0.228526 & -2.135133 \\
C8A & 7.235344 & 0.117738 & -1.199896 \\
H8A & 7.773542 & 0.211582 & -2.137288 \\
C10A & -3.148015 & -2.485316 & 0.421638 \\
C11A & -1.880829 & -3.289751 & 0.098177 \\
H11D & -1.628847 & -3.213750 & -0.961353 \\
H11E & -2.037382 & -4.343291 & 0.343099 \\
H11F & -1.032667 & -2.927833 & 0.680532 \\
C12A & -4.317347 & -3.067252 & -0.390681 \\
H12D & -5.275687 & -2.618865 & -0.131748 \\
H12E & -4.392245 & -4.140117 & -0.193471 \\
H12F & -4.150006 & -2.934898 & -1.462436 \\
C13A & -3.437300 & -2.581007 & 1.931391 \\
H13D & -2.611769 & -2.157055 & 2.507197 \\
H13E & -3.560138 & -3.626444 & 2.229259 \\
H13F & -4.355772 & -2.052301 & 2.195883 \\
C16A & -5.842871 & 0.121255 & 1.201545 \\
H16A & -5.301780 & 0.228779 & 2.135098 \\
C17A & -7.235345 & 0.117857 & 1.199863 \\
H17A & -7.773540 & 0.211789 & 2.137247 \\
\hline
\end{tabular}




\section{(S4). Solid-state NMR}

Solid-state NMR spectra were recorded at temperatures of $5-10{ }^{\circ} \mathrm{C}$ on a $9.4 \mathrm{~T}\left(400 \mathrm{MHz}{ }^{1} \mathrm{H}\right.$ Larmor frequency) wide-bore instrument. $\mathbf{3}$ and $\mathbf{4}$ were prepared in micro-crystalline form and packed under inert atmosphere into 2.5-mm and 4.0-mm MAS rotors. ${ }^{1} \mathrm{H}-{ }^{29} \mathrm{Si}$ and ${ }^{1} \mathrm{H}-{ }^{13} \mathrm{C}$ cross-polarization $(\mathrm{CP})$ spectra were recorded on 4.0-mm rotors, with MAS frequencies of $1675 \mathrm{~Hz}, 2860 \mathrm{~Hz}$ and $7000 \mathrm{~Hz}$ for ${ }^{29} \mathrm{Si}$, and $11000 \mathrm{~Hz}$ for ${ }^{13} \mathrm{C} .{ }^{1} \mathrm{H}-{ }^{31} \mathrm{P} \mathrm{CP}$ spectra were recorded on 2.5-mm rotors at MAS frequencies of $5700 \mathrm{~Hz}, 7000 \mathrm{~Hz}$ and $20000 \mathrm{~Hz}$. For 4, the $7 \mathrm{kHz}{ }^{1} \mathrm{H}-{ }^{31} \mathrm{P}$ spectra were recorded on a $4.0-\mathrm{mm}$ rotor.

The swept-frequency TPPM sequence was used for proton decoupling during acquisition, with an RF field amplitude of $83.3 \mathrm{kHz}$, sweep window of 0.32 and phase angle of $12.5^{\circ} .^{\text {[S8] }}$ Chemical shifts were calibrated using external references: TMS for ${ }^{29} \mathrm{Si}, 1 \mathrm{M} \mathrm{H}_{3} \mathrm{PO}_{4}$ for ${ }^{31} \mathrm{P}$, adamantane for ${ }^{13} \mathrm{C}$ (corresponding to DSS at $0 \mathrm{ppm}$ ).

The chemical shift anisotropies of ${ }^{29} \mathrm{Si}$ and ${ }^{31} \mathrm{P}$ were extracted by comparison of spinning sideband intensities with the sideband pattern of simulated spectra (Figure S4.2). Simulated spectra were generated within the numerical simulation routine $\mathrm{GAMMA}^{[\mathrm{S} 9]}$ over a large range of possible anisotropy and asymmetry values. The ${ }^{29} \mathrm{Si}$ or ${ }^{31} \mathrm{P}$ anisotropic chemical shift was considered as the relevant internal system Hamiltonian, and powder averaging involved 1154 crystallite orientations. Values which minimized the peak intensity residual sum of squares were used to produce the back-calculated spectra (Table S4.1). 
Table S4.1. Chemical shift tensor parameters and optimum CP contact times

\begin{tabular}{cccrrrrrr}
\hline Compound & Nucleus & $\begin{array}{c}\tau_{\mathrm{CP}} \\
(\mathrm{ms})\end{array}$ & $\begin{array}{c}\delta_{\text {iso }} \\
(\mathrm{ppm})\end{array}$ & $\begin{array}{c}\delta_{\mathrm{xx}} \\
(\mathrm{ppm})\end{array}$ & $\begin{array}{c}\delta_{\mathrm{yy}} \\
(\mathrm{ppm})\end{array}$ & $\begin{array}{c}\delta_{\text {zz }} \\
(\mathrm{ppm})\end{array}$ & $\eta_{\text {asym }}$ & \multicolumn{1}{c}{$\begin{array}{c}\delta_{\text {aniso }} \\
(\mathrm{ppm})\end{array}$} \\
\hline $\mathbf{3}$ (exp.) & ${ }^{31} \mathrm{P}$ & 5.6 & -326.1 & -367.7 & -367.7 & -242.9 & 0 & 83.2 \\
\hline $\mathbf{3}$ (theo.) & ${ }^{31} \mathrm{P}$ & & -341.1 & -405.6 & -389.9 & -227.9 & 0.139 & 113.2 \\
\hline $\mathbf{4}$ (exp.) & ${ }^{31} \mathrm{P}$ & 11.6 & -166.4 & -49.6 & -63.4 & -386.2 & 0.063 & -219.8 \\
\hline $\mathbf{4}$ (theo.) & ${ }^{31} \mathrm{P}$ & & -132.0 & 39.7 & -43.0 & -392.8 & 0.317 & -260.8 \\
\hline $\mathbf{3}$ & ${ }^{29} \mathrm{Si}$ & 5.1 & -8.4 & 43.8 & 3.4 & -72.4 & 0.631 & -64.0 \\
\hline $\mathbf{4}$ & ${ }^{29} \mathrm{Si}$ & 7.8 & 24.0 & -46.2 & -21.8 & 140.0 & 0.210 & 116.0 \\
\hline
\end{tabular}

The following convention is employed:

The principal components of the CSA tensor are $\delta_{\mathrm{xx}}, \delta_{\mathrm{yy}}$ and $\delta_{\mathrm{zz}}$, with $\left|\delta_{\text {zz }}-\delta_{\text {iso }}\right| \geq\left|\delta_{\text {xx }}-\delta_{\text {iso }}\right| \geq\left|\delta_{\text {yy }}-\delta_{\text {iso }}\right|$

Isotropic chemical shift: $\delta_{\text {iso }}=\frac{1}{3}\left(\delta_{\mathrm{xx}}+\delta_{\mathrm{yy}}+\delta_{\mathrm{zz}}\right)$

Anisotropy: $\delta_{\text {aniso }}=\delta_{\text {zz }}-\delta_{\text {iso }}$

Asymmetry: $\eta_{\text {asym }}=\frac{\delta_{\mathrm{yy}}-\delta_{\mathrm{xx}}}{\delta_{\text {aniso }}}$, where $\eta_{\text {asym }}=0$ is axially symmetric;

$\tau_{\mathrm{CP}}$ is the cross-polarization contact time yielding maximum intensity. 
a

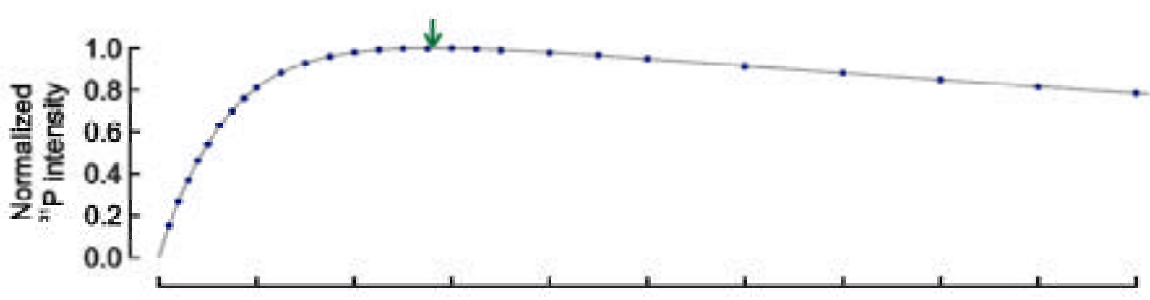

b

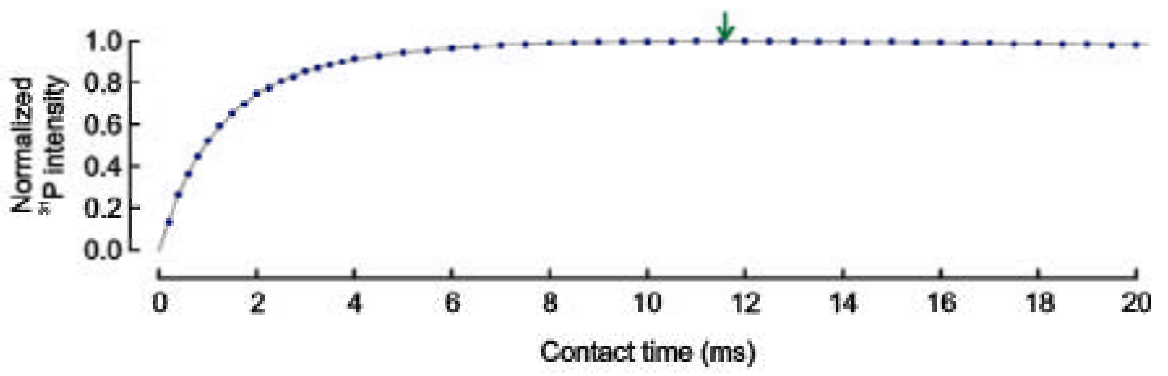

c

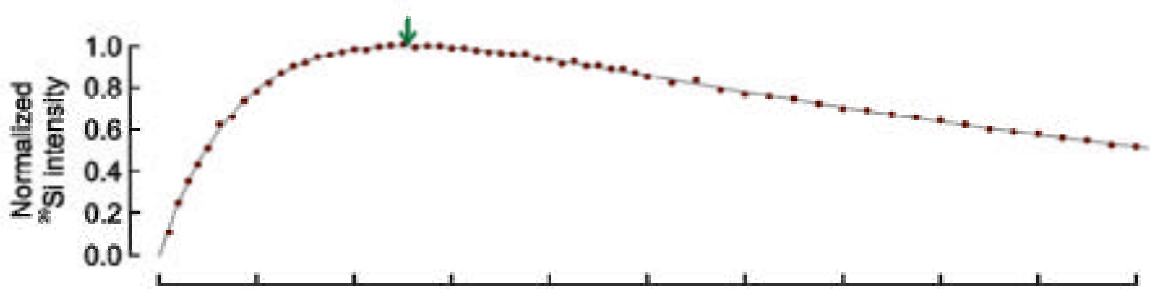

d

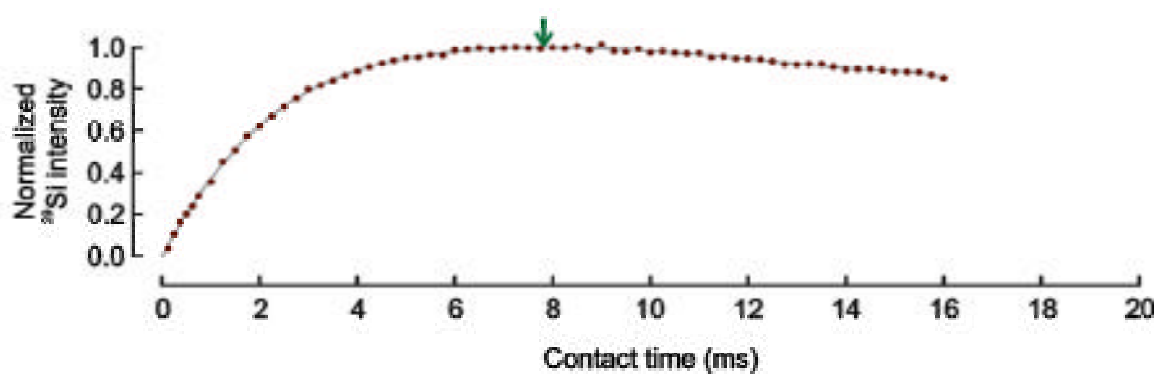

Figure S4.1. Cross-polarization profiles. The build-up of magnetization for compounds 3 (a,c) and $4(b, d)$ was monitored by incrementing the contact time between ${ }^{1} \mathrm{H}$ and ${ }^{31} \mathrm{P}(\mathrm{a}, \mathrm{b}$; $20 \mathrm{kHz}$ MAS), or ${ }^{1} \mathrm{H}$ and ${ }^{29} \mathrm{Si}$ (c,d; $7 \mathrm{kHz}$ MAS). Arrows indicate the point of maximal intensity. 

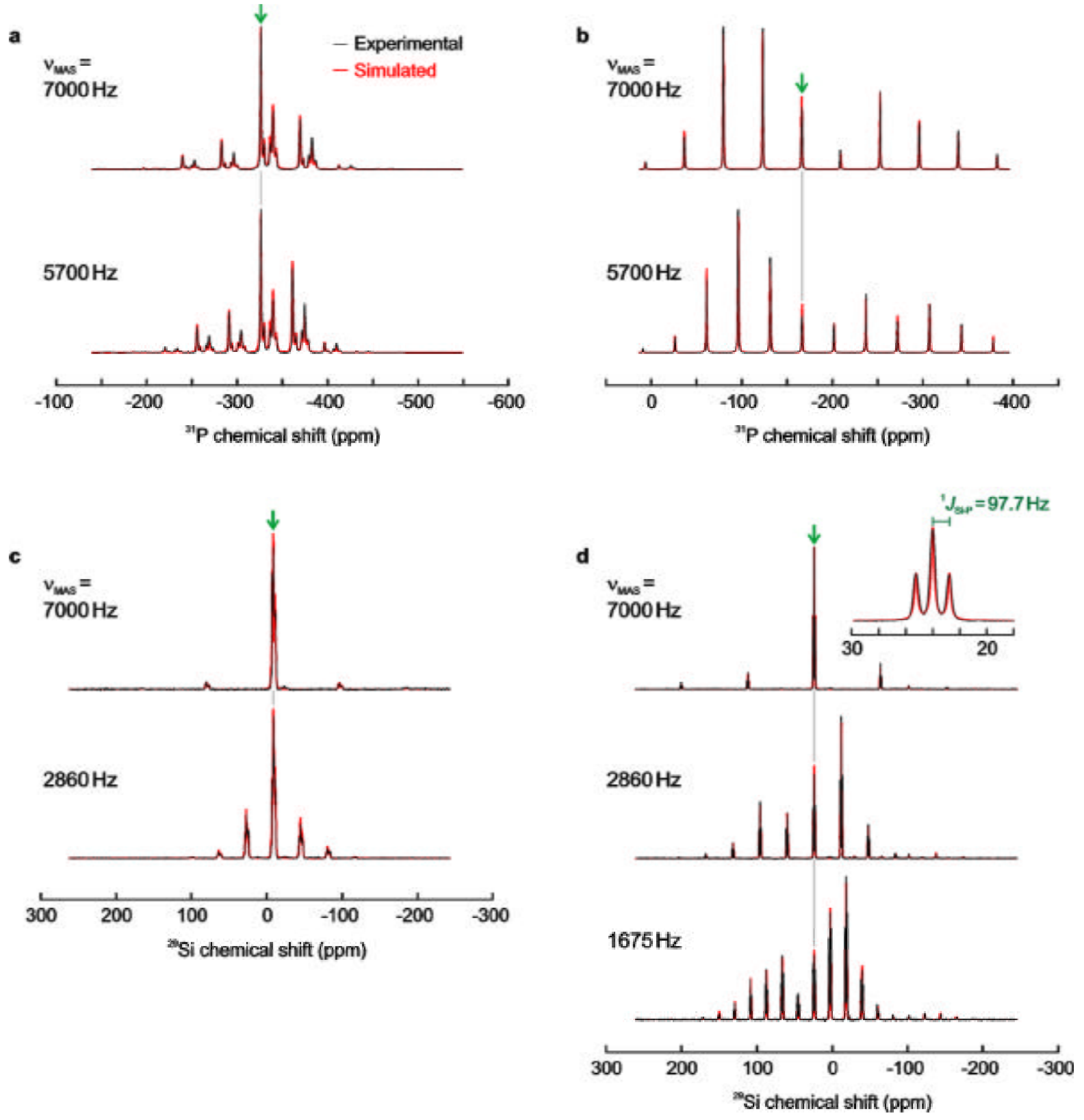

Figure S4.2. Experimental (black) and simulated (red) spinning sideband patterns for compounds 3 (a,c) and 4 (b,d). Cross-polarization spectra were recorded on ${ }^{31} \mathrm{P}(\mathrm{a}, \mathrm{b})$ and ${ }^{29} \mathrm{Si}$ $(\mathrm{c}, \mathrm{d})$ at multiple magic-angle spinning frequencies $\left(\mathrm{v}_{\mathrm{MAS}}\right)$. The isotropic peaks are indicated by arrows. The excerpt in (d) presents the multiplet structure of the ${ }^{29} \mathrm{Si}$ resonance of $\mathbf{4}$ due to silicon-phosphorus $J$ coupling. 
(S5). Tentative mechanism for the formation of 3 and 4 from 2.

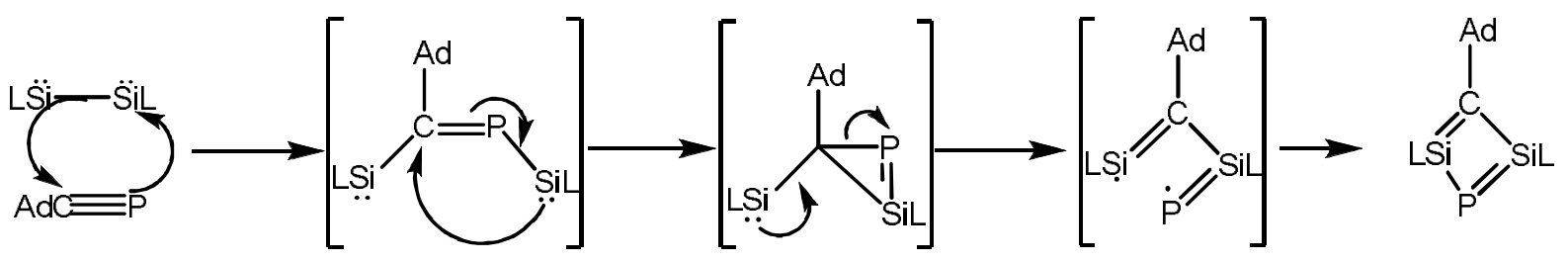

Mechanism for the formation of $\mathbf{3}$

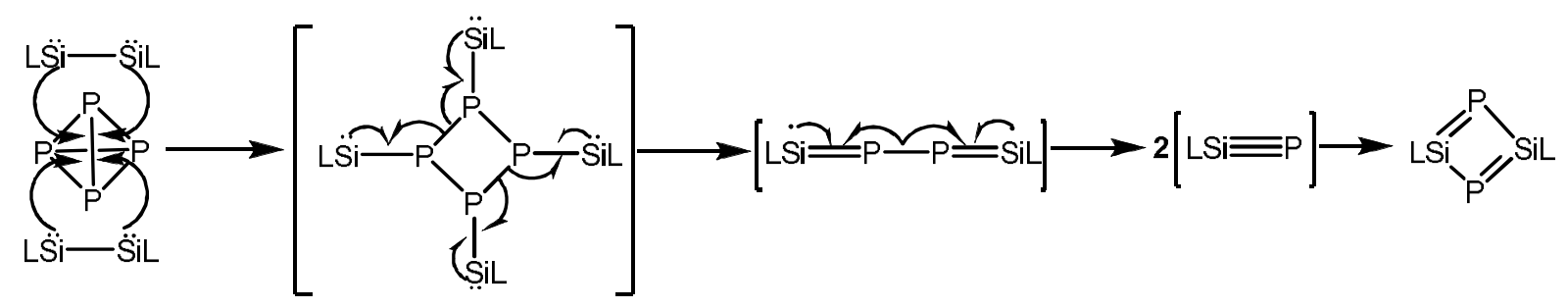

Mechanism for the formation of 4

(S6). References

[S1] S. S. Sen, H. W. Roesky, D. Stern, J. Henn, D. Stalke, J. Am. Chem. Soc. 2010, 132, $1123-1126$.

[S2] S. S. Sen, A. Jana, H. W. Roesky, C. Schulzke, Angew. Chem. 2009, 121, 8688 8690; Angew. Chem. Int. Ed. 2009, 48, 8536 - 8538.

[S3] T. Kottke, D. Stalke, J. Appl. Crystallogr. 1993, 26, 615 - 619.

[S4] Bruker, SAINT v 7.68A., Bruker AXS Inc., Madison (WI, USA), 2009.

[S5] G. M. Sheldrick, SADABS 2008/2, Göttingen, 2008.

[S6] G. M. Sheldrick, Acta Crystallogr. Sect. A 2008, 64, 112 - 122.

[S7] M. J. Frisch, G. W. Trucks, H. B. Schlegel, G. E. Scuseria, M. A. Robb, J. R. Cheeseman, G. Scalmani, V. Barone, B. Mennucci, G. A. Petersson, H. Nakatsuji, M. Caricato, X. Li, H. P. Hratchian, A. F. Izmaylov, J. Bloino, G. Zheng, J. L. Sonnenberg, M. Hada, M. Ehara, K. Toyota, R. Fukuda, J. Hasegawa, M. Ishida, T. Nakajima, Y. Honda, O. Kitao, H. Nakai, T. Vreven, J. A. Montgomery, Jr., J. E. Peralta, F. Ogliaro, M. Bearpark, J. J. Heyd, E. Brothers, K. N. Kudin, V. N. Staroverov, R. Kobayashi, J. Normand, K. Raghavachari, A. Rendell, J. C. Burant, S. 
S. Iyengar, J. Tomasi, M. Cossi, N. Rega, J. M. Millam, M. Klene, J. E. Knox, J. B. Cross, V. Bakken, C. Adamo, J. Jaramillo, R. Gomperts, R. E. Stratmann, O. Yazyev, A. J. Austin, R. Cammi, C. Pomelli, J. W. Ochterski, R. L. Martin, K. Morokuma, V. G. Zakrzewski, G. A. Voth, P. Salvador, J. J. Dannenberg, S. Dapprich, A. D. Daniels, O. Farkas, J. B. Foresman, J. V. Ortiz, J. Cioslowski and D. J. Fox, Gaussian, Inc., Wallingford CT, 2009.

[S8] a) C. V. Chandran, P. K. Madhu, N.D. Kurur, T. Bräuniger, Magn. Reson. Chem., 2008, 46, 943 - 947; b) R. S. Thakura, N. D. Kurur, P. K. Madhu, Chem. Phys. Lett., 2006, $426,459-463$

[S9] S.A. Smith, T.O. Levante, B.H. Meier, R.R. Ernst, J. Magn. Reson. A, 1994, 106, 75 105. 